\title{
Etude du rôle de la microstructure sur les propriétés électromagnétiques du fer ex-carbonyle
}

\author{
D. ROUSSELLE et F. RAVEL
}

Service Matériaux Avances, CEA/DAM, Centre d'Etudes de Bruyeres-le-Chateh BP. 12, 91680 Bruyeres-le-Chatel, France

\begin{abstract}
Thermal decomposition of pentacarbonyl iron is known to produce spherical micronic iron particles. By this process, special microstructure, called "onion skin structure", is obtained. We try to correlate this structure with powder composition especially with the impurities (such as carbon, nitrogen and oxygen) which have been introduced during the elaboration process. Then, the influence of this onion skin structure on the electromagnetic properties of iron carbonyl powder has been investigated. Thermal annealings have been carried out at various temperatures under different atmospheres (hydrogen,argon) and the induced effects on microstructure and impurities contents have been studied. $300^{\circ} \mathrm{C}$ annealing in flowing $\mathrm{H}_{2}$ resulted in a sensible growth of cristallites, as shown by XRD and MET studies, and $450^{\circ} \mathrm{C}$ annealing in a complete disappearance of the onion skin structure. Concerning microwaves properties, annealed samples exhibited a lower resonance frequency and a lower intrinsic permeability, deduced from effective medium approximation. These samples were compared to non carbonyl iron powders so as to explain this behaviour.
\end{abstract}

\section{INTRODUCTION}

Le procédé d'élaboration de poudre de fer par décomposition du fer pentacarbonyle $\mathrm{Fe}(\mathrm{CO})_{s}$, connu depuis 1920 ,permet de synthétiser des particules de fer de forme sphérique et de taille micronique [1]. Le principe est le suivant:

$\mathrm{Fe}(\mathrm{CO})_{5}$, vaporisé à une température proche de son point d'ébullition $\left(105^{\circ} \mathrm{C}\right)$, est introduit au sommet d'une chambre de décomposition dans laquelle la température varie de 250 a $450^{\circ} \mathrm{C}$. Il se forme alors des nuclei de fer sur la surface desquels le fer pentacarbonyle continue à se décomposer. L'azote, issu de l'ammoniac introduit dans le but de contrôler la germination, et le carbone, résultant de la décomposition de $\mathrm{Fe}(\mathrm{CO})_{5}$, sont les principales impuretés présentes dans la poudre ainsi élaborée.

Dans le domaine des matériaux absorbants micro-ondes, le fer ex-carbonyle est souvent utilisé pour ses propriêtés électromagnétiques en tant que charge dispersée dans une matrice amagnétique isolante. Le comportement en hyperfréquences du composite ainsi formé peut se décrire à partir du modèle de gyromagnétisme amorti, établi par LANDAU et 
LIFSHITZ[2]. Il est directement lié aux caractéristiques magnétiques de la charge, et dépend donc de la microstructure des particules de fer ex-carbonyle .

Nous avons étudié plus particulièrement le grade EQ commercialisé par BASF, pour lequel nous avons cherché d'abord à préciser sa microstructure, puis à la faire évoluer par l'intermédiaire de traitements thermiques afin de comprendre les résultats obtenus en hyperfréquences.

\section{TECHNIQUES EXPERIMENTALES.}

Les caractéristiques microstructurales des poudres ont été obtenues à partir des clichés réalisés par microscopie optique après attaque au nital, par microscopie électronique en transmission ( Philips CM12 ) après découpe préalable au microtome, et par diffraction de rayons $X$ sur un diffractomètre Siemens D500 équipé d'une anticathode de cuivre $(\lambda=$ $1.5406 \AA$ ).

Les compositions chimiques ont été mesurées par analyse de gaz dans les métaux et par fluorescence $X$.

Les poudres de fer ex-carbonyle EQ ont été traitées thermiquement sous balayage d'hydrogène dans un dispositif de tube toumant, afin d'éviter l'agrégation des particules, a 150,300 et $450^{\circ} \mathrm{C}$ pendant 3 heures.

Pour comparer la perméabilité magnétique $\mu$ et la permittivité $\varepsilon$ des différents échantillons, nous avons incorporé les poudres dans une résine Epoxy avec des taux volumiques de charge voisins de $50 \%$. Les mesures de $\mu$ et $\varepsilon$ ont été réalisées en ligne coaxiale APC7 avec un analyseur de réseau Hewlett-Packard 8510 de $50 \mathrm{MHz}$ à $18 \mathrm{GHz}$.

\section{RESULTATS}

\section{1.MICROSTRUCTURE DE LA POUDRE AVANT TRAITEMENT THERMIQUE}

Avant traitement thermique, la poudre de fer ex-carbonyle présente une microstructure originale en pelure d'oignon (Fig. 1) correspondant à des couches concentriques d'épaisseur 0,1 a $0,4 \mu \mathrm{m}$ de contraste différent.

Sur le spectre réalisé par diffraction des rayons X (Fig. 2), la forme Fe- $\alpha$ est prédominante et accompagnée d'impuretés semblant correspondre au carbure de fer hexagonal $\mathrm{Fe}_{2} \mathrm{C}-\varepsilon$. On constate également une largeur relativement importante des raies qui peut être attribuée à une faible taille des cristallites et/ou à la présence de fortes contraintes dans le réseau. Cette faible taille est confirmée par les clichés en MET (Fig.3) qui montrent des cristallites de l'ordre de $10 \mathrm{~nm}$. On décèle sur cliché de diffraction électronique la présence du carbure $\mathrm{Fe}_{3} \mathrm{C}$. Ce résultat n'est pas incompatible avec ceux obtenus par diffraction des rayons $\mathrm{X}$ car, sous l'effet du faisceau électronique, la phase $\mathrm{Fe}_{2} \mathrm{C}$ est susceptible de s'être transformée en $\mathrm{Fe}_{3} \mathrm{C}$. 


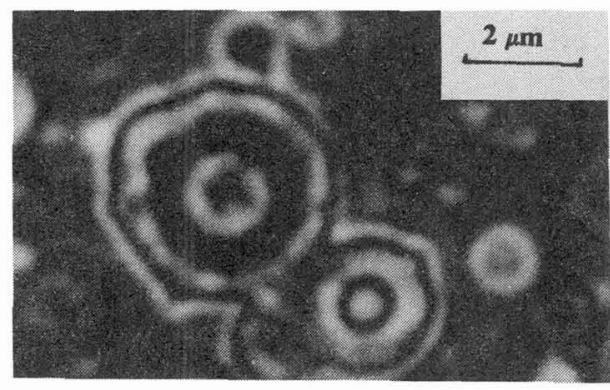

Figure 1 : Micrographie de la structure en pelure d'oignon de la poudre de fer $E Q$.

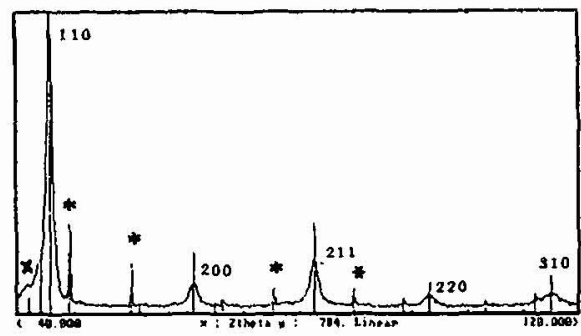

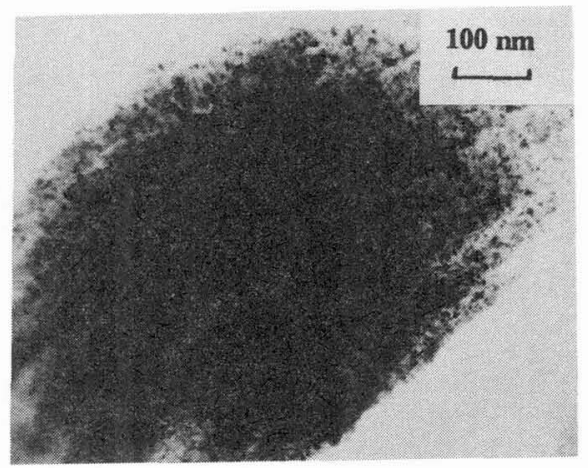

Figure 3: Cliché de MET en fond clair montrant la taille des cristallites de fer $a$.

$$
\begin{aligned}
& \text { raies indextes }=\text { fer } \alpha \\
& *=\text { étalon silicium } \\
& x=\text { carbure } \mathrm{Fe}_{2} \mathrm{C}
\end{aligned}
$$

Eigure 2:Spectre de diffraction de RX de la poudre avant traitement thermique.

\section{II.2.EVOLUTION DE LA COMPOSITION CHIMIQUE.}

Le tableau I présente les teneurs en impuretés en fonction de la température de traitement thermique.

Tableau I: Composition chimique en ppm des poudres traitées.

\begin{tabular}{|c|c|c|c|c|}
\cline { 2 - 5 } \multicolumn{1}{c|}{} & non traité & $150{ }^{\circ} \mathrm{C}$ & $300^{\circ} \mathrm{C}$ & $450^{\circ} \mathrm{C}$ \\
\hline oxygène & $3400 \pm 340$ & $3540 \pm 180$ & $2250 \pm 110$ & $2170 \pm 110$ \\
\hline azote & $11600 \pm 1200$ & $8630 \pm 430$ & $25 \pm 5$ & $30 \pm 5$ \\
\hline carbone & $10000 \pm 1000$ & $8840 \pm 440$ & $80 \pm 5$ & $40 \pm 5$ \\
\hline
\end{tabular}

Les traitements thermiques sous hydrogène, dès $300{ }^{\circ} \mathrm{C}$, ont eu pour effet de diminuer nettement la teneur en carbone et azote. Plus la température est élevée, plus la teneur en oxygène est réduite mais elle reste toutefois relativement importante (diminution de $36 \%$ par rapport à l'échantillon non traité pour un recuit à $450^{\circ} \mathrm{C}$ ).

\section{II.3.EVOLUTION DES LARGEURS DE RAIES DE DIFFRACTION.}

A partir des largeurs de raies $\beta_{\mathrm{hkl}}$ mesurées, on peut tracer la $\operatorname{courbe} \beta \cos \theta=\mathrm{f}(\sin \theta)$ dont l'ordonnée à l'origine permet d'évaluer la taille des cristallites et la pente la valeur moyenne des contraintes [3]. La figure 4 nous donne l'évolution de ces deux paramètres en fonction de la température du recuit. 


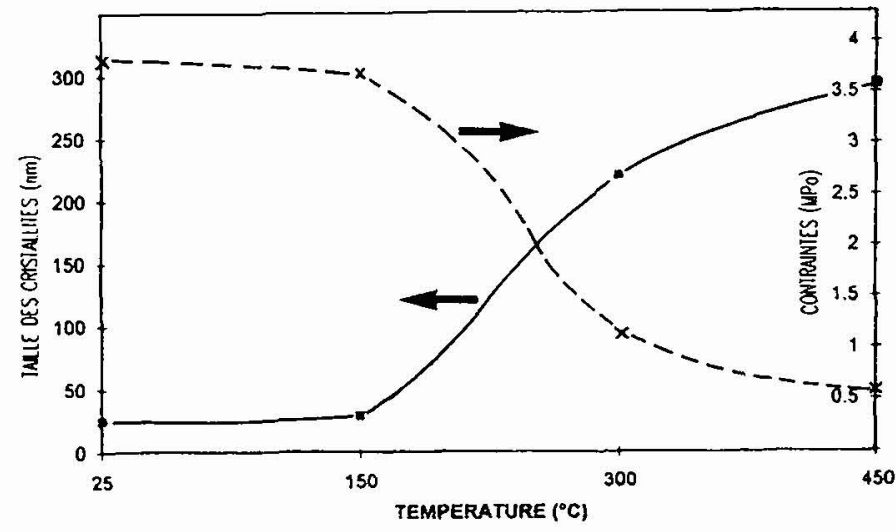

Figure 4: Evolution de la taille des cristallites et des contraintes en fonction de la température de traitement thermique.

On retrouve pour 1'échantillon non traité une taille des cristallites voisine de celle observée en MET. Un recuit à $300{ }^{\circ} \mathrm{C}$ suffit à faire augmenter fortement cette taille et entraîne également une relaxation des contraintes.

II.4. EVOLUTION DE LA STRUCTURE EN PELURE D'OIGNON.

A partir de $300{ }^{\circ} \mathrm{C}$, la morphologie de type pelure d'oignon commence à disparaître pour devenir totalement absente dans le cas de l'échantillon recuit a $450^{\circ} \mathrm{C}$ pendant 3 heures (Fig.5).
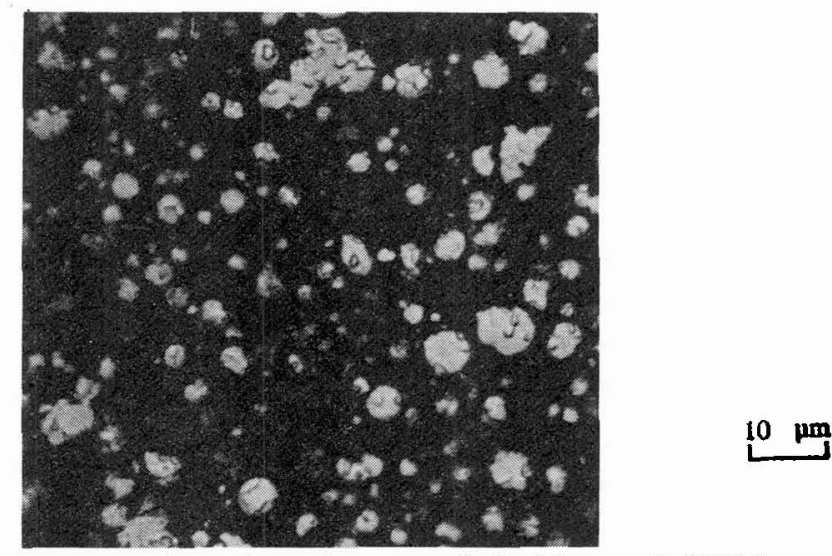

Eigure 5: Microstructure observée après un recuit de 3 heures à $450^{\circ} \mathrm{C}$ sous $\mathrm{H}_{2}$.

\section{II.5.EVOLUTION DES PROPRIETES MAGNETIQUES,}

Le tableau II présente les caractéristiques magnétiques statiques en fonction de la température du recuit. L'aimantation à saturation des échantillons augmente avec la température du traitement thermique. Cette variation est reliée directement à la réduction du taux d'impuretés. 
Tableau II: Aimantation à saturation et champ coercitif des différents échantillons.

\begin{tabular}{|c|c|c|c|c|}
\cline { 2 - 5 } \multicolumn{1}{c|}{} & non traité & $150^{\circ} \mathrm{C}$ & $300^{\circ} \mathrm{C}$ & $450^{\circ} \mathrm{C}$ \\
\hline$\sigma_{\mathrm{S}}($ uem/g) & 201,2 & 201,6 & 210,3 & 211,7 \\
\hline $\mathbf{H}_{\mathrm{c}}(\mathrm{Oe})$ & $50,0 \pm 2,2$ & $57,0 \pm 2,3$ & $45,0 \pm 1,7$ & $58,0 \pm 2,6$ \\
\hline
\end{tabular}

\section{II.6. EVOLUTION DES PROPRIETES MAGNETIQUES INTRINSEQUES}

La théorie de milieu effectif de Bruggeman [4] permet d'exprimer la perméabilité effective du composite $\left(\mu_{\mathrm{e}}\right)$, accessible par la mesure, en fonction de la perméabilité de la charge $\left(\mu_{\mathrm{j}}\right)$ et de la matrice $\left(\mu_{\mathrm{m}}\right)$ et de leurs concentrations respectives (c et 1-c). Dans ce modele, on considère que les particules baignent dans le milieu moyen recherché. Dans le cas d'inclusions sphériques, l'équation de Bruggeman s'écrit:

$$
c \frac{\mu_{\mathrm{i}}-\mu_{\mathrm{e}}}{\mu_{\mathrm{i}}+2 \mu_{\mathrm{e}}}+(1-\mathrm{c}) \frac{\mu_{\mathrm{m}}-\mu_{\mathrm{e}}}{\mu_{\mathrm{m}}+2 \mu_{\mathrm{e}}}=0
$$

Il est alors facile d'extraire la perméabilité intrinsèque de la charge suivant l'équation:

$$
\mu_{\mathrm{i}}=\mu_{\mathrm{e}} \frac{2 \mu_{\mathrm{e}}+3 \mathrm{c}-2}{\mu_{\mathrm{e}}(3 \mathrm{c}-1)+1}
$$

L'application de cette loi aux différents échantillons [5] montre une évolution de la perméabilité intrinsèque à partir de $30{ }^{\circ} \mathrm{C}$ (figure 6): la fréquence de résonance est légèrement plus faible et la perméabilité imaginaire correspondante plus basse que dans le cas de l'échantillon non traité. On remarque, de plus, que la valeur de $\mu$ " à basse fréquence dessous $\mathrm{du} \mathrm{GHz}$ ) augmente avec la température de recuit. Le comportement magnétique intrinsèque d'une poudre de fer obtenue par électrolyse (Fig.7), de même granulométrie que le fer "EQ" mais présentant une microstructure classique, est analogue (même fréquence de résonance mais amplitude inférieure) à celui de l'échantillon recuit à $450^{\circ} \mathrm{C}$ pour lequel la pelure d'oignon a completement disparu. Enfin, on peut également noter que la valeur de la perméabilité statique intrinsèque du fer ex-carbonyle obtenue par inversion de la loi de BRUGGEMAN est voisine de 20 , ce qui est en accord avec la valeur de 22.7 de VEINBERG [6]. 


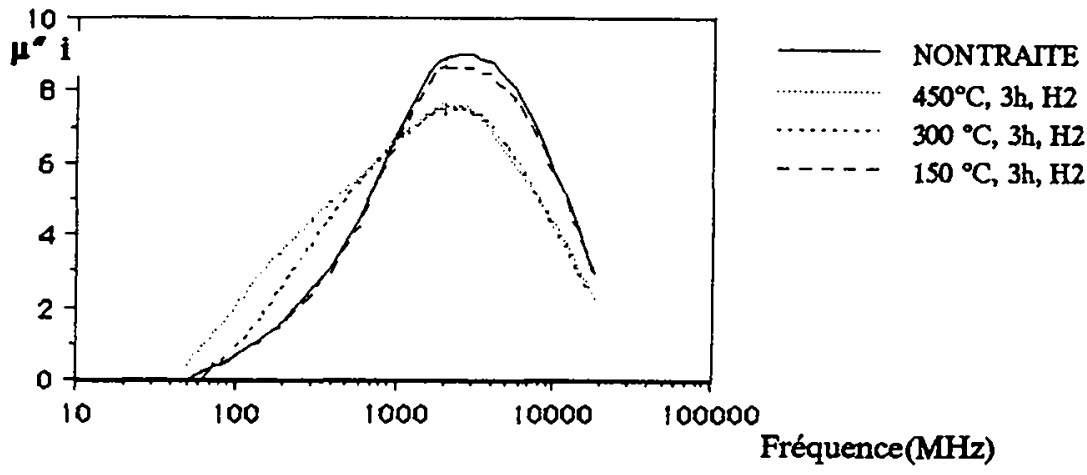

Figure 6: Influence de la température du traitement thermique.

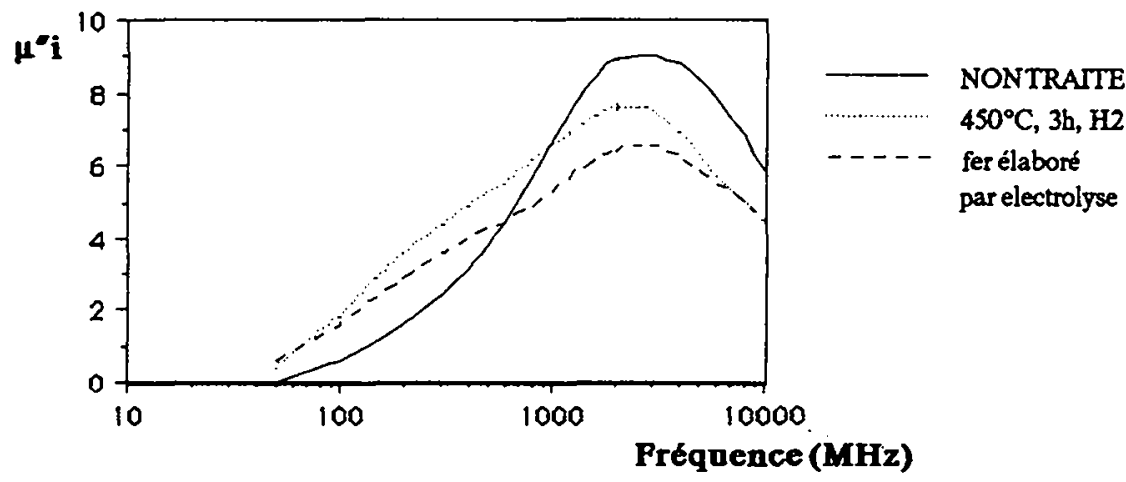

Figure 7: Comparaison avec une poudre de fer élaborée par un autre procédé.

\section{III.DISCUSSION.}

La microstructure de la poudre issue de la décomposition du fer pentacarbonyle présente un caractère instable. La phase de carbure décelée sur la poudre brute disparaît dès $300{ }^{\circ} \mathrm{C}$, améliorant ainsi les propriêtés magnétiques statiques (aimantation à saturation). Cette disparition s'accompagne d'un changement de microstructure (élimination de la "pelure d'oignon") associé à une relaxation des contraintes ainsi qu'une croissance importante des cristallites. De plus, l'évolution de la microstructure a pour effet de modifier le comportement magnétique dynamique. On observe une augmentation de $\mu$ " dans la gamme $50 \mathrm{MHz}-1 \mathrm{GHz}$ que l'on peut attribuer à l'apparition d'une résonance de parois rendue possible par la forte croissance des cristallites (Tableau III).

On observe également une légère modification de la fréquence de résonance gyromagnétique qui peut s'expliquer, d'après la formule de Kittel, par une diminution du champ d'anisotropie due au recuit. Cette évolution semble compatible avec la relaxation des contraintes déduite de l'analyse cristallographique.

Enfin, la diminution de $\mu^{\prime \prime}{ }_{\max }$ après recuit pourrait provenir de l'apparition d'un effet de peau: l'épaisseur de peau, déduite de la formule classique en prenant pour résistivité la 
valeur theorique du fer, est de l'ordre du diametre des particules. Lorsque la microstructure est du type "pelure d'oignon", la pénétration de l'onde électromagnétique semble favorisée par la succession de couches concentriques de faible épaisseur. Au contraire, quand on fait disparaître cette microstructure par des traitements thermiques, on accentue l'effet de peau. La densité de matière magnétique effective diminue conduisant ainsi à des niveaux de perméabilité plus faibles.

Tableau III: Corrélation entre $\mu^{n}$ à $200 \mathrm{MHz}$ et la taille des cristallites

\begin{tabular}{|c|c|c|}
\hline Température $\left({ }^{\circ} \mathrm{C}\right)$ & $\mu^{\prime \prime}$ intrinsèque à $200 \mathrm{MHz}$ & taille des cristallites $(\AA)$ \\
\hline non traité & 1,6 & 250 \\
\hline 150 & 1,5 & 300 \\
\hline 300 & 2,8 & 2200 \\
\hline 450 & 3,2 & 2940 \\
\hline
\end{tabular}

\section{CONCLUSION}

Nous avons cherché à corréler la microstructure en "pelure d'oignon" du fer ex-carbonyle avec les impuretés ( $C, N, O)$. Des moyens de caractérisation (Rayons X, MEB, MET) nous ont permis de mettre en évidence des cristallites de fer $a$ très contraints de l'ordre de la dizaine de nanomètres, ainsi que la présence de précipités très fins de carbure de fer $\mathrm{Fe}_{2} \mathrm{C}$. Nous avons montré que, à partir d'un type de charge, il est possible d'ajuster le niveau de perméabilité et la fréquence de résonance du matériau composite correspondant simplement en faisant évoluer la microstructure des particules par des recuits à une température modérée. La structure en "pelure d'oignon" du fer ex-carbonyle n'a pas encore livré tous ses secrets. Pour affiner la compréhension, des caractérisations plus poussées (Rayons X en température et EXAFS) ont été entreprises.

\section{REMERCIEMENTS}

Les auteurs tiennent à remercier $P$. Brault et $C$. Boscher pour leur participation aux expérimentations, C.Dalle, M.Leibbrandt et L.Autissier pour l'analyse radiocristallographique, J.Y.Boixière et N.Bardy pour les mesures magnétiques, M.Capelle et F.Espert pour les micrographies, et M.Caput et J.Ramolfo pour les clichés de MET.

\section{BIBLIOGRAPHIE}

[1] TAYLOR A, Soft Magnetic Materials for Telecommunications, New York,Interscience Publishers Inc.(1953).

[2] LANDAU L, D, LIFSCHITZ E, M, Electrodynamics of continuous media Pergamon Press (1984).

[3] MEGAW,STOKES, Proc. Roy. Soc. 61(1948)382.

[4] BRUGGEMAN D, A, Ann. Physik, Leipzig 11(1880)70.

[5] ROUSSELLE D. These Universite de BORDEAUX I 1992.

[6] VEINBERG A, K, Sov. Phys. Dolk, 11(1967)593. 